\title{
Editorial
}

\section{Percutaneous Surgical Revolution for Vertebral Compression Fractures}

\author{
Hossam Elnoamany ${ }^{1} \quad$ Mohamed Adel $^{1}$ \\ ${ }^{1}$ Department of Neurosurgery, Menoufia University Hospital, \\ Faculty of Medicine, Menoufia, Egypt \\ Indian J Neurosurg:2020;9:73-74
}

The vertebral column plays two major roles: first is a structural, weight-bearing role as the centerpiece of the axial skeleton and second as the conduit for the spinal cord. The lumbar and lower thoracic vertebrae are larger and wider as required for their weight-bearing role and as supports for the upper body and axial skeleton.

Many clinical entities can cause painful vertebral collapse. These include osteoporosis, vertebral hemangiomas, multiple myelomas, and metastatic diseases.

Vertebral compression fractures (VCFs) of the thoracic and lumbar spine are usually associated with major trauma and can cause spinal cord damage that result in neural deficits. This usually results in an anterior wedge compression fracture. As the name implies, the anterior column is compressed, with sometimes varying degrees of middle and posterior column insult.

Many surgical treatment strategies had been employed to treat such fractures either by open surgery or minimally invasive ones. Percutaneous vertebroplasty was first introduced by Galibert in 1987 for the treatment of vertebral angiomas, and after few years, he started treating VCFs by the same technique. ${ }^{1}$ Percutaneous vertebroplasty was indicated for vertebral compression fractures with intact posterior vertebral body border and when the patient is complaining of back pain without neural deficit.

Percutaneous vertebroplasty considered at that time as first-generation vertebral cementing augmentation to fix VCFs resulting from traumatic insult, osteoporosis, and vertebral metastasis.

There were many drawbacks for this technique such as frequent cement leak with resultant neural compression, due to a large amount of cement injected under high pressure and inability to correct the kyphotic deformity secondary to VCFs; therefore, this technique was considered by some surgeons as only a pain relief surgical technique.

For me, by some modification on the cement injection technique, which leads to injection of serial small amounts of cement into the affected vertebra, surgeons had minimized the risk of cement leak significantly. ${ }^{2}$

By year 2000, Wong invented the balloon kyphoplasty which considered second-generation vertebroplasty. ${ }^{3}$ In this technique, the surgeon will make a pathway transpedicular into the fractured vertebra using a Gamshidi needle. A small balloon is then guided through the needle into the bone. Once in position, the balloon is slowly inflated to gently raise the collapsed bone into its normal position. When the bone is in the correct position, the surgeon deflates the balloon and removes it. This leaves behind a cavity within the vertebral body. To prevent the bone from collapsing again, the cavity is filled with cement. The cement forms a cast inside the vertebral body which stabilizes the bone. To fully secure the bone, the procedure is sometimes performed on both sides of the vertebral body.

Despite good results achieved from balloon kyphoplasty with respect to incidence of cement leak and correction of kyphotic deformity, the overall clinical results showed no significant superiority over first-generation vertebroplasty. ${ }^{4}$

Unlike the VCFs resulting from trauma or osteoporosis, the vertebral osteolytic lesions from metastasis were treated by percutaneous selective vertebroplasty. In this technique, the surgeon can achieve dual benefits by aspirating the tumor tissue and then injecting cement under low-pressure to support the affected vertebra. ${ }^{2}$

Rupture of balloon intracorporial during inflation process had been experienced, inability of inflated balloon to restore vertebral height in old VCFs, and adjacent level fractures due to large amount of hard injected cement, made the minimally invasive spine surgeons community to search for more stable devices that can treat kyphotic angle and need less amount of injected cement to stabilize the affected vertebra.

Third-generation vertebroplasty devices have been introduced lately. First, the vertebral body stenting; then, OsseoFix vertebral implant and Kiva vertebral device. Finally, Spinejack vertebral augmentation device have been used to treat VCFs. ${ }^{5}$

The vertebral body stent is an expandable titanium device for percutaneous vertebral augmentation. It aims to alleviate pain and restore the height of the fractured vertebra. This system uses the same balloon, as in a standard kyphoplasty, which is inserted and expanded into the vertebral body. This device produces a concrete reduction of the fracture, thus recovering the vertebral height and providing a cavity into which highly
C2020 Neurological Surgeons' Society of India
License terms

()(1) $\Theta \circledast$ 
viscous cement can be injected. Thereafter, the balloon can be deflated and removed without risk of partial height loss because the device remains inside the vertebral body.

OsseoFix is an expandable titanium mesh which is introduced via transpedicular approach to expand into the vertebral body, with the aim of reducing the vertebral fractures and decreasing the kyphotic deformity by compacting the surrounding trabecular bone. This device can be used alone or with cement.

As far as Kiva is concerned, it is a flexible continuous loop-like vertebral implant. Using a unilateral percutaneous transpedicular approach, the implant is inserted in affected vertebra and bone cement is injected through and inside the implant. Kiva offers many potential benefits, including reduced rate of adjacent level fracture, improved kyphotic angle restoration, reduced rate of bone cement leakage, and reduced amount of bone cement needed.

SpineJack is a new titanium device designed to restore the height of the vertebral body. A bilateral percutaneous transpedicular approach with fluoroscopic guidance is used to insert the SpineJack device into the vertebral body under the most caudal part of the collapse. Expansion of the device applies a 500 Newton distraction force to the fracture, along the craniocaudal axis, similar to a jack. Device expansion is achieved using a specific tool that pulls the two ends of the implant toward each other, shortening the device and deploying the central titanium component. A rack-and-pinion system blocks the expansion of the implant at the desired height, while preventing any loss of correction before the injection of cement, which envelops the implants, ensuring definitive stabilization of the fracture. The use of two devices symmetrically positioned inside the vertebral body allows a homogeneous spreading of the cement.

Spinejack vertebral implant is a mechanical system which has a direct lifting mechanism. That is why it is suitable for treating both recent and old VCFs. It reduces the rate of adjacent levels fracture by reducing cement volume, improves kyphotic angle restoration, decreases rate of cement leak, and reduces acute pain, achieving significant clinical improvement over balloon kyphoplasty.

In our practice, we treat VCF patients with first-generation vertebroplasty if their MRI shows bone marrow edema, and loss of anterior body height is less than $30 \%$. As cement injection alone can stabilize the affected vertebra, and the height can be restored to the extent that the angle of kyphosis can be corrected.

However, in patients with VCFs not associated with bone marrow edema in their MRI or where the loss of anterior body height is more than $30 \%$, we use SpineJack third-generation vertebroplasty, if available. If not available, balloon kyphoplasty second-generation vertebroplasty can be used.

\section{Conflicts of Interest}

None declared.

\section{References}

1 Galibert P, Deramond H, Rosat P, Le Gars D. Preliminary note on treatment of vertebral angioma by percutaneous acrylic vertebroplasty. Neurochirgie 1978;33:166-168

2 Elnoamany H. Percutaneous selective vertebroplasty: state of the art management in well-confined metastatic vertebral lesions. Asian Spine J 2016;10(5):869-876

3 Theodorou DJ, Theodorou SJ, Duncan TD, Garfin SR, Wong WH. Percutaneous balloon kyphoplasty for the correction of spinal deformity in painful vertebral body compression fractures. Clin Imaging 2002;26(1):1-5

4 Xing D, Ma J-X, Ma X-L, et al. A meta-analysis of balloon kyphoplasty compared to percutaneous vertebroplasty for treating osteoporotic vertebral compression fractures. J Clin Neurosci 2013;20(6):795-803

5 Vanni D, Galzio R, Kazakova A, et al. Third-generation percutaneous vertebral augmentation systems. J Spine Surg 2016;2(1):13-20 